Decay of Surface Nanostructures via Long-Time-Scale Dyanmics

Author(s):

Arthur F. Voter, Nikolai Stanciu $\mathrm{T}-12$

Los Alamos National Laboratory, an affirmative action/equal opportunity employer, is operated. by the University of California for the U.S. Department of Energy under contract W-7405-ENG-36. By acceptance of this article, the publisher recognizes that the U.S.

Government retains a nonexclusive, royalty-free license to publish or reproduce the published form of this contribution, or to allow others to do so, for U.S. Government purposes. Los Alamos National Laboratory requests that the publisher identify this article as work performed under the auspices of the U.S. Department of Energy. The Los Alamos National Laboratory strongly supports academic freedom and a researcher's right to publish; as an institution, however, the Laboratory does not endorse the viewpoint of a publication or guarantee its technical correctness. 


\section{DISCLAIMER}

This report was prepared as an account of work sponsored by an agency of the United States Government. Neither the United States Government nor any agency thereof, nor any of their employees, makes any warranty, express or implied, or assumes any legal liability or responsibility for the accuracy, completeness, or usefulness of any information, apparatus, product, or process disclosed, or represents that its use would not infringe privately owned rights. Reference herein to any specific commercial product, process, or service by trade name, trademark, manufacturer, or otherwise does not necessarily constitute or imply its endorsement, recommendation, or favoring by the United States Government or any agency thereof. The views and opinions of authors expressed herein do not necessarily state or reflect those of the United States Government or any agency thereof. 


\section{DISCLAIMER}

Portions of this document may be illegible in electronic image products. Images are produced from the best available original document. 


\title{
Decay of Surface Nanostructures via Long-Time-Scale Dyanmics
}

\author{
Arthur F. Voter* and Nikolai Stanciu
}

\begin{abstract}
This is the final report of a three-year. Laboratory Directed Research and Development (LDRD) project at the Los Alamos National Laboratory (LANL). We have developed a new approach for extending the time scale of molecular dynamics simulations. For "infrequent-event" systems, the category that includes most diffusive events in the solid phase, this "hyperdynamics" method can extend the simulation time by a few orders of magnitude compared to direct molecular dynamics. The trajectory is run on a potential surface that has been biased to raise the energy in the potential basins without affecting the transition state region. The method is described and applied to surface and bulk diffusion processes, achieving microsecond and millisecond simulation times. We have also developed a new parallel computing method that is efficient for small system sizes. The combination of the hyperdynamics with this parallel replica dynamics looks promising as a general materials simulation tool.
\end{abstract}

\section{Background and Research Objectives}

Atomistic simulations are playing an increasingly important role in chemistry and materials science. Developments in empirical potentials, dynamical methods, and computer speed have all contributed to the power of simulations in addressing real problems that were insoluble just ten years ago. Molecular dynamics (MD) simulations can access times up to tens of nanoseconds for systems with thousands of atoms. On a large parallel computer, millions of atoms can be simulated. This time-scale is long enough to investigate phenomena such as surface sputtering [1], surface and bulk self-diffusion at high temperature [2], surface melting [3], ion-implant cascades [4], and high-strain-rate crack propagation [5].

For activated processes with identifiable transition states, this nanosecond timescale can be extended considerably using transition state theory (TST) and dynamical corrections [6,7]. For example, this allows one to study simple surface and bulk diffusion mechanisms. For more complex processes, such as diffusion within a submonolayer overlayer on an ideal crystal surface, it is sometimes possible to compute barrier heights for all possible environments that can influence an atomic jump event, and use this catalog of

*Principal Investigator, e-mail: afv@lanl.gov 
rate constants in a stochastic algorithm known as kinetic Monte Carlo [8]. An example is shown in Figure 1. If all diffusion mechanisms are known, this method gives the exact dynamics for the state-to-state evolution of the system. This approach has been applied, for example, to overlayer self-diffusion on $\mathrm{Ag}(100)$ [9] and $\mathrm{Si}(100)$ [10], and is gaining acceptance for simulating dopant and defect diffusion in semiconductors.

Despite these advances, however, there remains a class of important problems that cannot be addressed with any of these methods. These problems are characterized by intrinsic time scales that greatly exceed the nanoseconds accessible to direct $\mathrm{MD}$, and a complexity that precludes the use of cataloged, kinetic Monte Carlo methods. In these systems the required catalog is prohibitively large and/or the dynamical evolution involves complicated, many-particle reaction coordinates that defy categorization and cannot be guessed in advance. Processes in this class include vapor-deposited film growth, diffusive motion of a dislocation, and annealing of crystal damage after ion implantation or neutron bombardment. For the surface deposition case, Figures 2 through 4 illustrate the problem. Even for the simplest possible metal surface (fcc(100)), our understanding of the adatom diffusion mechanism was overturned as recently as 1990 [11]. The mechanisms discovered since then (see Figure4) are numerous and complex, and must shake our confidence about whether the picture is complete, even now. Although the dynamical evolution can be accelerated by raising the temperature of the simulation, in general this causes the system to proceed along different pathways. For example, the growth mode of a metal crystal during vapor deposition can change from layer-by-layer to three-dimensional growth and then back to layer-by-layer as the temperature is raised, due to the delicate interplay of the different diffusion barriers [12].

The primary goal of this project was to develop a general computational method for solving problems of this type, without appealing to kinetic Monte Carlo. The concept was that by using a direct dynamical approach, with a built-in accelerator of some sort, the requirement of specifying all the possible escape paths could be eliminated. This method would have the character of a regular molecular dynamics trajectory, which "finds" its way out of a potential energy basin without knowing anything in advance about the possible escape paths. It was not clear how the accelerator would work, but something based on importance sampling concepts seemed plausible, and the payoff seemed high.

To increase the probability of success in the event that this primary goal was too ambitious, two other more modest goals were also stated in the original proposal. The first of these (the secondary goal) was to develop a general saddle-point seeking algorithm that could be used to extend the rate-driven kinetic Monte Carlo method to more complex systems. The other (the tertiary goal) was to study the time-dependent decay of novel 
surface nanostructures. Surface nanostructures were also proposed as the test systems to use in developing the new methods stated in the primary and secondary goals, as their dynamics exhibit the complexity that makes study with kinetic Monte Carlo difficult or impossible.

Despite its high-risk nature, the three years of research emphasized the primary goal and, happily, success was achieved. The resulting new method, termed "hyperdynamics," is described below, with a discussion of its strengths, weaknesses, and the types of processes for which it is well suited. A companion approach, which harnesses the power of parallel computers to extend the time scale of small systems, is also described. The development of this "parallel replica" method occurred near the end of the 3-year LDRD cycle, and was funded in part by the Office of Basic Energy Sciences of the Department of Energy. As the emphasis (and success) of this project involved the primary goal, the secondary and tertiary goals are not discussed further.

\section{Importance to LANL's Science and Technology Base and National R\&D Needs}

The capability to extend the time scale of molecular dynamics simulations beyond the nanosecond range has value in a number of areas of technological importance to Los Alamos, the Department of Energy, and the nation. Materials deformation involves processes such as crack tip evolution (either blunting or extension), dislocation motion, and stress-assisted diffusion of point defects and defect clusters. In real experiments, these processes typically occur on time scales that are inaccessible to direct molecular dynamics simulation. A description of many of these processes on the microsecond and millisecond time scales (and perhaps beyond) should be possible using the new approaches described here. This will improve our understanding of materials failure, processing, and aging, and therefore impact energy efficiency, weapon integrity, and structural materials design.

The controlled deposition of metallic thin films is a critical step in the manufacture of computer integrated circuits. The methods developed in this project take us a large step closer to being able to simulate the metal film growth process at a realistic growth rate. Two major U.S. integrated circuit manufacturers will soon be applying these new methods to better understand the metal deposition processing step.

Accurate simulation of radiation damage events and the microstructural evolution that occurs after an event are important to the nuclear industry and other industries where ion implantation is used as a processing tool. The time-scales involved in the annealing stage far exceed that available to direct molecular dynamics, and the methods developed here look promising for making progress on this problem. The Office of Basic Energy Sciences of DOE is now funding the further development of hyperdynamics and parallel- 
replica dynamics to study radiation damage annealing, beginning with copper as a prototype.

\section{Scientific Approach and Accomplishments Infrequent Event Systems}

As discussed above, the goal is to develop a method for extending the time available to molecular dynamics (MD) simulation. We focus on the category of "infrequent event" systems, in which the trajectory spends the vast majority of its time simply vibrating in a basin of the potential energy surface. Occasionally, it finds an escape path, making a transition to a new potential basin by passing over the ridge top separating the two states (this ridge-top boundary is known as the dividing surface). For a short time following this initial escape, the system still has additional energy localized in the reaction coordinate, and may make additional crossings of this same dividing surface, or may pass through another dividing surface to yet another state. These are referred to as correlated dynamical events. For example, an atom diffusing on a surface will sometimes make a double jump, rather than simply hopping to the nearest-neighbor site. Nonetheless, after a brief time (a couple of picoseconds for a metal), the excess energy dissipates into other vibrational modes in the system, and the trajectory settles into some potential basin, losing all memory of how it arrived in that state. We will refer to this time until memory is lost as the correlation time. The large disparity between the correlation time and the average time between transitions is what defines an infrequent event system. This separation of time scales also gives rise to some useful statistical properties, which are exploited to derive the two methods.

\section{Transition State Theory}

Assuming that one knows the position of this ridge-top dividing surface surrounding a state, a good approximation to the escape rate can be calculated without ever running a trajectory, by appealing to transition state theory (TST). In the TST approximation, the rate constant is taken as the outgoing flux through the dividing surface. This is an equilibrium property of the system, depending on the shape and energy of the many-dimensional basin, and on the shape and energy of the ridge top. If the system never executes correlated dynamical events (i.e., if every time a trajectory crosses a dividing surface, it settles down in the new state without any recrossings), the TST approximation is exact. For diffusive processes in materials, TST is an excellent approximation; typically the correlated dynamical events cause only a few-percent error in the rate constant. With the TST approach, the average time until an escape event occurs can be computed directly, even if this escape time is much longer than the feasible simulation time for a trajectory. 
In situations where the exact rate constant is desired, it can be obtained by computing a dynamical correction factor that multiplies the TST rate constant [6,7]. This correction factor is straightforward to calculate from the results of trajectories that are initiated at the dividing surface (with properly Boltzmann-sampled starting points) and evolved for a time a bit longer than the correlation time. An appealing aspect of computing the rate constant in this way is that the product of the TST rate with the dynamical correction factor gives the exact rate constant regardless of the location of the dividing surface. Thus, for example, if the dividing surface does not lie along the peak of the ridge, the TST rate constant will increase, but the dynamical correction factor will decrease to compensate. That is, some trajectories that pass through this "bad" dividing surface (which contribute to the flux, and hence the TST rate) will not actually make it over the ridge top. These trajectories that fall back into the initial state contribute negatively to the dynamical correction factor. Thus, transition state theory and dynamical corrections theory make a robust framework from which to develop alternative approaches for simulating the dynamical evolution in a many-state system. Perhaps surprisingly, this even allows construction of methods that accelerate the dynamical evolution of systems in which the locations of the dividing surfaces are unknown.

\section{Hyperdynamics Method}

In the hyperdynamics method, we assume that there are no correlated recrossing events -- i.e., that TST is exact. As mentioned above, this is an excellent approximation for the types of materials processes we wish to study. The basic concept is to use the properties of transition state theory to define a modified dynamical system that gives accelerated transitions from state to state. We derive it qualitatively here, while the full derivation can be found in Reference [13] (Publication 1).

Consider a trajectory in a basin (state A) of a potential energy surface. It wanders around in this basin for many vibrational periods (perhaps thousands or millions) until it happens to find the dividing surface that leads to an adjacent state. From transition state theory, we know that the average time until it finds this surface is simply a property of the shape and energy of the dividing surface relative to the shape and energy of the basin of state A. More precisely, it is proportional to the ratio of the partition function of the $3 \mathrm{~N}-1$ dimensional dividing surface to the partition function of the $3 \mathrm{~N}$ dimensional basin. If we modify the basin of state $\mathrm{A}$ to have less configuration space, thus lowering its partition function, we raise the escape rate in a predictable way.

A schematic example of this type of modification is shown in Figure 5. The energy of the state-A basin has been raised by adding a bias potential. In addition, the basins of states $B$ and $C$ have been raised, as have the other states of the system. Note that we have 
taken care not to change the energy along the dividing surfaces. This is a requirement of the hyperdynamics method, and a key point is that we can accomplish this even when we do not know in advance where the dividing surfaces may be. This is demonstrated below. The other requirement on the bias potential is that it preserve the TST-obeying nature of the dynamics; i.e., whether a trajectory is run on the original potential surface or on the biased potential surface, no correlated recrossing events can occur.

A dynamical simulation on a surface modified in this way has two desirable properties. First, the escape rate from each state is enhanced. This is obvious, as the wells are not as deep as for the original potential. Second, the relative rates of escape from state $A$ to the various adjacent states ( $\mathrm{B}$ and $\mathrm{C}$ in this case) are not affected. This is not so obvious, but results from the fact that each rate is a ratio of the dividing surface partition function to the basin partition function. Taking the ratio of two rates thus eliminates any dependence on the basin itself, leaving only the ratio of dividing surface partition functions. The consequence of these two properties is that a trajectory run on this surface will make transitions at an accelerated rate, and will execute a sequence of state-to-state transitions that is indistinguishable from that of a long trajectory on the original potential surface. The details of the dynamical behavior within any of the basins are irretrievably changed by the bias potential, but this is a sacrifice we happily make to achieve the accelerated transition rate.

The simulation time scale, which is accelerated in a nonlinear fashion (the bias potential will, in general, increase the escape rates more for some states than for others), can be recovered from an extremely simple procedure. At each integration step during the trajectory, the hyper-MD (or "boosted time") clock is advanced by the normal MD time step $\left(\Delta \mathrm{t}_{\mathrm{MD}}\right.$ ) multiplied by the inverse Boltzmann factor for the strength of the bias potential at that point in configuration-space, i.e., $\Delta \mathrm{t}_{\text {hyper }}=\Delta \mathrm{t}_{\mathrm{MD}} \exp \left(\Delta \mathrm{V} / \mathrm{k}_{\mathrm{B}} \mathrm{T}\right)$. In the course of a simulation, this instantaneous boost factor fluctuates wildly, but over a long run, gives a well-defined average boost factor. This definition for the boosted time converges on the exact time as the simulation runs, even though the system may escape from each state long before adequate sampling of the bias potential is obtained, and even if every state of the system is different [13].

To summarize the hyperdynamics formalism: assuming a bias potential can be constructed that raises the potential basins without affecting the dividing surfaces, a trajectory run on this biased potential surface evolves from state to state in a proper sequence. Time is no longer an independent variable, but is instead estimated statistically as the simulation runs, in a way that gives a vanishing relative error at long simulation times. In contrast to a kinetic Monte Carlo approach, hyperdynamics has the simplicity of a 
regular molecular dynamics simulation. It wanders around in each potential basin until it accidentally passes through a dividing surface into another state. Despite not knowing anything about the other possible escape routes, it picks one path with the correct Boltzmann probability.

This hyperdynamics formalism is only useful if it is possible to design a computationally efficient bias potential that is zero at the dividing surfaces without advanced knowledge of their location. In principle, the exact position of a dividing surface can be determined by following steepest-descent paths downhill from a number of different geometries. The dividing surface separates those paths that fall into one basin from those that fall into another basin. While this tedious procedure is mathematically well defined, we want a simpler definition, and one that can tell us how far the trajectory is from a dividing surface, so that the bias potential can be turned off smoothly as the dividing surface is approached. This type of definition can be created using the local slope and curvature properties of the potential surface. Specifically, the gradient vector gives the slope of the surface, and the matrix of second derivatives (the Hessian matrix) gives the curvature information.

As an example, consider the two-dimensional model potential shown in Figure 6. (The form of this potential, and the details of this calculation, are given in [13].) In this case, the dividing surface is the vertical line defined by $x=0$. At any point on the potential surface, there are two principle curvatures, which can be found by diagonalizing the Hessian matrix. Because by definition the lowest principle curvature (the lowest eigenvalue) is negative at any saddle point, a relatively simple definition for the bias potential is one that smoothly turns on as the lowest eigenvalue of the Hessian goes positive, and is zero whenever it is negative. The result of this simple approach is shown in Figure 7. While the potential near the minimum is altered significantly, the dividing surface is hardly affected. Figure 8 shows the evolution of the boosted time for a trajectory on this biased potential surface at a reduced temperature of 0.1 for a short section of hyperdynamics trajectory. Notice that it evolves very nonlinearly, indicating that the trajectory moves in and out of the regions with the high bias potential. At this temperature, the average boost factor is 3435 . Figure 9 shows an Arrhenius plot of the diffusion constants computed at a number of temperatures. The average boost values (shown next to each point) are seen to increase as the temperature is lowered. This is a general characteristic of this approach, resulting from the inverse Boltzmann form of the instantaneous boost factor. The hyperdynamics results agree perfectly with those from direct molecular dynamics. At the lower temperatures, direct MD is unfeasible, but the 
exact dynamical result can be computed using dynamically corrected TST [14], as discussed above.

For higher-dimensional systems, such a simple form for the bias potential is inadequate, because the Hessian has all positive eigenvalues in a vanishingly small fraction of the total space. Thus, for realistic systems, we use a more complicated function of the gradient and Hessian information, by exploiting an approximate, local definition for the dividing surface position proposed by Sevick, Bell, and Theodorou [15]. Their definition is exact at the saddle points of the system, and a good approximation nearby. This expression, and its incorporation into a form that defines a smooth bias potential, is described in Reference [16] (Publication 2). In this approach, one must choose a value for the strength of the bias potential that is lower than the lowest barrier in the system. In real applications, where the mechanisms and barriers are not known, the lowest barrier can be estimated from the length of time the simulation has run without any observed event. Although the bias potential in this approach depends formally on the second derivatives of the potential (and the forces needed for MD would thus depend on the third derivatives), an iterative algorithm [16] allows propagation of the hyperdynamics trajectory using only first derivatives, just as for normal molecular dynamics. The necessary quantities are accumulated by making a few dozen evaluations of the interatomic forces at positions very close to the instantaneous position of the trajectory. In applications so far using embedded atom interatomic potentials (a good description for fcc transition metals [17]), this procedure has been found to require about 20 to 50 times as much work per hyperMD step as a regular MD step would.

In a hyperdynamics simulation of $\mathrm{Ag}$ adatom diffusion on $\mathrm{Ag}(100)$ at $\mathrm{T}=400 \mathrm{~K}$, both hopping and exchange events were observed, and the average boost factor was 1356 . The extra work per computational step (compared to normal MD) was about 30 times. Thus, the same simulation ( 9.89 microseconds total boosted time) performed with direct MD would have required $1356 / 30=45$ times more computational work. During this 9.89 microseconds, 23 hops and 16 exchanges were observed, corresponding to rate constants that agree with the correct values, estimated from full harmonic transition state theory [18] (direct molecular dynamics was unfeasible). For this simulation, the bias potential strength was set to $0.3 \mathrm{eV}$ based on the known barrier heights, which are slightly above $0.5 \mathrm{eV}$.

Figure 10 shows an application of hyperdynamics to a more complex system, the diffusive motion of a 10-atom $\mathrm{Ag}$ cluster on the $\mathrm{Ag}(111)$ surface [16]. Using a bias potential with parameters similar to those employed in the $\mathrm{Ag} / \mathrm{Ag}(100)$ case, and allowing two substrate layers to move (for a total of 70 moving atoms), a boost factor of 8310 was obtained at a cost of about 20 times the work per integration step. In a few weeks of CPU 
time on a scientific workstation, the system evolved for 221.2 microseconds. The nonlinear evolution of the boosted time is shown in Figure 11. The cluster does not move far in this time, but we can observe a number of complicated, concerted mechanisms that might be omitted from a kinetic Monte Carlo simulation. For example, at 9.5 microseconds, all 10 atoms jump in the same direction to move the cluster from hcp to fcc registry (Figures. 10a and 10b), a mechanism that was unknown until 1992 [19]. At 94.5 microseconds, the cluster breaks out of its compact configuration, via a mechanism analogous to the dimer-shearing on $\mathrm{fcc}(100)$ [20], allowing adatom motion at the cluster periphery (Figures. 10c-10f). At 197.8 microseconds (Figures. 10g-10i), there is a dislocation event [21], resulting in a rotation of the cluster orientation.

This example serves to illustrate the generality of the hyperdynamics approach and the time scales accessible with it. While for this system all the observed events (and perhaps their relative frequencies) could have been predicted based on extrapolation downward from direct MD simulations at higher temperatures, such an extrapolation becomes impossible for a system as complex as a growing surface. Hyperdynamics should be helpful for understanding these more complicated systems, on time scales approaching those that occur between deposition events. With further development, direct simulation of realistic deposition rates may be possible in certain cases, especially when used in conjunction with the new parallel method described below.

Tests so far have given us some sense of the strengths and weaknesses of the hyperdynamics method. These are in turn guiding the application to systems where it can have the most impact, and showing us what future developments are most important. Due to the way the bias potential is currently designed, the method works best for systems that have tight vibrational coupling. Metallic surface diffusion is well described, as is diffusion in the bulk. For this reason, we are currently focusing on metallic film growth and annealing of radiation damage in solids. In the near future, we will apply hyperdynamics to ionic solids, which we expect will also work well. On the other hand, floppy molecules adsorbed on a surface tend to give very low boost factors, presumably because the bias function gets "confused" by the strongly anharmonic floppy modes, thinking it is seeing dividing surfaces everywhere. Diffusion in a polymeric solid has this same characteristic. A system-specific bias potential may offer a way around this, though this has not been attempted yet.

Systems with low barriers are also problematic. This is because the bias potential can only be turned on safely to a level that remains below the lowest barrier. For example, diffusion of a single adatom on a fcc(111) metal surface has a barrier below $0.1 \mathrm{eV}$, so that the hyperdynamics boost is very low (well below break-even) at room temperature. When 
there is a mix of low and high barriers, it is possible that intentional blocking of the lowbarrier pathways can offer access to the higher energy pathways at higher boost levels. This type of modification must be performed very carefully to prevent corrupting the dynamics. We are beginning to study this problem.

Because the average boost factor will be large for systems in which the lowest barrier is high relative to the temperature, hyperdynamics is best employed on systems where a regular molecular dynamics simulation can reach many nanoseconds. Another way to state this is that activated processes in most physical systems have a standard preexponential factor of about one inverse picosecond. Even if the temperature is set very high, the events will not occur any faster than about once per picosecond. Similarly, if the bias potential is so effective that the well depth is reduced to zero, the rate will be increased to once per picosecond. The greater the ratio between the time between events that can be accessed with regular MD and this limiting time between events, the greater the possible boost factor. A consequence of this argument is that hyperdynamics is most effective when applied to small systems, with simple potentials, on fast computers. (In fact, if the hyperdynamics method existed 20 years ago when computers were much slower, there would be no point in using it. It would run slower than regular MD.)

Because the hyperdynamics method is more effective on faster computers, harnessing parallel processing to make the simulation run even faster would be ideal. However, existing parallel molecular dynamics algorithms work by subdividing physical space, assigning the atoms within each region to a single processor. For systems smaller than about 1000 atoms, the surface-to-volume ratio of each subspace is so large that communication time dominates and the efficiency is severely degraded. For infrequent event systems, we have developed a new way to organize the work that circumvents this problem. We describe this next.

\section{Parallel Replica Method}

The "parallel replica" method [22] (Publication 5) works as follows for a computer with $M$ processors. On each processor, an independent trajectory for the entire system is propagated, while monitoring for a transition. Transitions can be efficiently detected using occasional steepest descent minimizations. When a transition to a new basin is detected (on processor $i$, say), the other processors are stopped, and the master simulation clock is advanced by the sum of the trajectory times accumulated on all the processors up to the time of the transition. The trajectory on processor $i$ is then continued for a short time (a picosecond or two) to allow completion of correlated dynamical events. This additional correlation time is added to the simulation clock, and the procedure is repeated by replicating the new geometry of processor $i$ onto all $M$ processors. This parallel replica 
method gives the correct probability distribution for the time between escapes, as shown in Figure 12 for the diffusion of a surface vacancy on $\mathrm{Cu}(100)$. It also preserves the relative probabilities of the different possible escape paths. The state-to-state dynamical evolution is thus exact, and proceeds nearly $M$ times faster than a single-processor trajectory, provided the average time between transitions is much longer than the time required to evolve the correlated events on one processor. Communication time is typically insignificant, although it could become important in implementations on hundreds of processors. If the trajectory on each processor is evolved using hyperdynamics instead of regular MD, a combined gain is observed. The overall computational gain is the average hyperdynamics boost factor, divided by the extra work per step compared to regular MD (typically $20-50 x$ ), multiplied by $M$, the number of processors.

We are currently testing this combined approach with the goal of developing a general tool for materials simulations. This effort is being funded in part by the Office of Basic Energy Sciences. Test runs on the Theoretical Division LOKI machine, which consists of 16 Pentium Pro processors, look promising. Figure 13 shows the simulation time plotted versus wall clock time for the initial stage of a simulation of bulk vacancy diffusion in silver. For comparison, the time scale resulting from regular molecular dynamics (on one processor), and the parallel replica method (16 times faster) are also shown. The bias strength in the hyperdynamics is ramped up slowly (beginning from zero -- i.e., regular MD), to prevent accidentally blocking an escape path. At regular time intervals, a new (higher) estimate is made of the lowest barrier in the system. This is calculated easily from the time (or hypertime) that has accumulated so far without any transition occurring. The bias strength is then increased to a value slightly below this new estimate. Although we know the escape paths in this relatively simple case, the goal is to test the viability and efficiency of this kind of completely "safe" approach. After an induction period of about one hour, the hyper+parallel time climbs exponentially away from the other curves. After the first transition occurs, the bias strength is reset to zero and the automatic bias strength procedure is started over again. In 65 hours, the simulation clock accumulated 1.9 milliseconds, and the vacancy made 10 diffusive jumps. This matches the correct rate constant, computed using the harmonic approximation [18]. In systems with lower barriers, the hyperdynamics boost is reduced considerably, but the parallel gain is still observed.

\section{Conclusion}

To conclude, the hyperdynamics method appears promising as a general atomistic simulation tool for processes in materials, especially metals. Classical trajectory simulations on the microsecond time scale are now possible for the first time. Combining 
hyperdynamics with the new parallel replica method makes it even more powerful, and this should be especially useful as parallel desktop systems become ubiquitous over the next few years. Further development is needed, especially for systems with floppy modes and low barriers.

\section{Publications}

1. Voter, A.F., "A Method for Accelerating the Molecular Dynamics Simulation of Infrequent Events," J. Chem. Phys., 106, 4665 (1997).

2. Voter, A.F., "Hyperdynamics: Accelerated Molecular Dynamics of Infrequent Events," Phys. Rev. Lett., 78, 3908 (1997).

3. Voter, A.F., "Accelerated Molecular Dynamics of Materials Defects with the Hyper-MD Method," in \{lit Novel Materials\}, edited by Rao, B.K., and Behera, S.N., in press (Nova Scientific, New York, 1997).

4. Voter, A.F., "Atomistic Simulation Methods," in \{Morphological Organizations in Epitaxial Growth and Removal \}, Zhang, Z. and Lagally, M., editors, in press (World Scientific, 1997).

5. Voter, A.F., "Parallel Replica Method for Dynamics of Infrequent Events," Phys. Rev. $\mathrm{B}$, in press. (submitted after end of LDRD program).

\section{References}

[1] Karetta, F. and Urbassek, H.M., "Molecular-dynamics simulations of bulk and surface damage production in low-energy $\mathrm{Cu}-\mathrm{Cu}$ bombardment," J. Appl. Phys. 71, 5410 (1992).

[2] Lyndenbell, R.M., "Migration of Adatoms on the (100) Surface of Face-Centred-Cubic Metals," Surf. Sci. 259,129 (1991).

[3] Chen, E.T., Barnett, R.N., and Landman, U., "Surface melting of Ni(110)," Phys. Rev. B 41, 439 (1990).

[4] De La Rubia, T.D., Caro, A., and Spaczer, M., "Kinetics of Radiation-Induced Disordering of A3B Intermetallic Compounds - A Molecular-Dynamics-Simulation Study," Phys. Rev. B 47, 11483 (1993).

[5] Zhong, W., Cai, Y., and Tomanek, D., "Computer Simulation of Hydrogen Embrittlement in Metals," Nature 362, 435 (1993).

[6] Voter, A.F. and Doll, J.D., "Dynamical Corrections to Transition State Theory for Multistate Systems: Surface Self-Diffusion in the Rare-Event Regime," J. Chem. Phys. 82, 80 (1985).

[7] Anderson, J.B., "Predicting rare events in molecular dynamics," Adv. Chem. Phys. 91, 381 (1995). 
[8] Voter, A.F., "Classically Exact Overlayer Dynamics: Diffusion of Rhodium Clusters on Rh(100)," Phys. Rev. B 34, 6819 (1986).

[9] Voter, A.F., "Simulation of the Layer-Growth Dynamics in Silver Films: Dynamics of Adatom Clusters and Vacancy Clusters on $\mathrm{Ag}(100)$, , in Modeling of Optical Thin Films, M.R. Jacobson, Ed., Proc. SPIE 821, 214 (1987).

[10] Lu, Y.T., Zhang, Z.Y., and Metiu, H., "The Migration of a Si Atom Adsorbed on the Si(100)-2x1 Surface," Surf. Sci. 257, 199 (1991).

[11] Feibelman, P.J., "Diffusion path for an $\mathrm{Al}$ adatom on $\mathrm{Al}(001)$," Phys. Rev. Lett. 65, 729 (1990).

[12] Egelhoff, W.F., Jr. and Jacob, I., "Reflection high-energy electron-diffraction (RHEED) oscillations at 77-K," Phys. Rev. Lett. 62, 921 (1989).

[13] Publication 1.

[14] Voter, A.F., Doll, J.D., and Cohen, J.M., "Using Multistate Dynamical Corrections to Compute Classically Exact Diffusion Constants at Arbitrary Temperature," J. Chem. Phys. 90, 2045 (1989).

[15] Sevick, E.M., Bell, A.T., and Theodorou, D.N., "A chain of states method for investigating infrequent event processes occuring in multistate, multidimensional systems," J. Chem. Phys. 98, 3196 (1993).

[16] Publication 2.

[17]Daw, M.S., Foiles, S.M., and Baskes, M.I., "The Embedded-Atom Method : A Review of Theory and Applications," Mater. Sci. Reports 9, 251 (1993).

[18] Vineyard, G.H., "Frequency Factors and Isotope Effects in Solid State Rate Processes," J. Phys. Chem. Solids 3, 121 (1957).

[19] Liu, C.L., and Adams, J.B., "Structure and diffusion of clusters on Ni surfaces" Surf. Sci. 268, 73 (1992).

[20] Shi, Z-P., Zhang, Z., Swan, A.K., and Wendelken, J.F., "Dimer shearing as a novel mechanism for cluster diffusion and dissociation on metal(100) surfaces," Phys. Rev. Lett. 76, 4927 (1996).

[21] Hamilton, J.C., Daw, M.S., and Foiles, S.M., "Dislocation mechanism for island diffusion on fcc (111) surfaces," Phys. Rev. Lett. 74, 2760 (1995).

[22] Publication 5. 


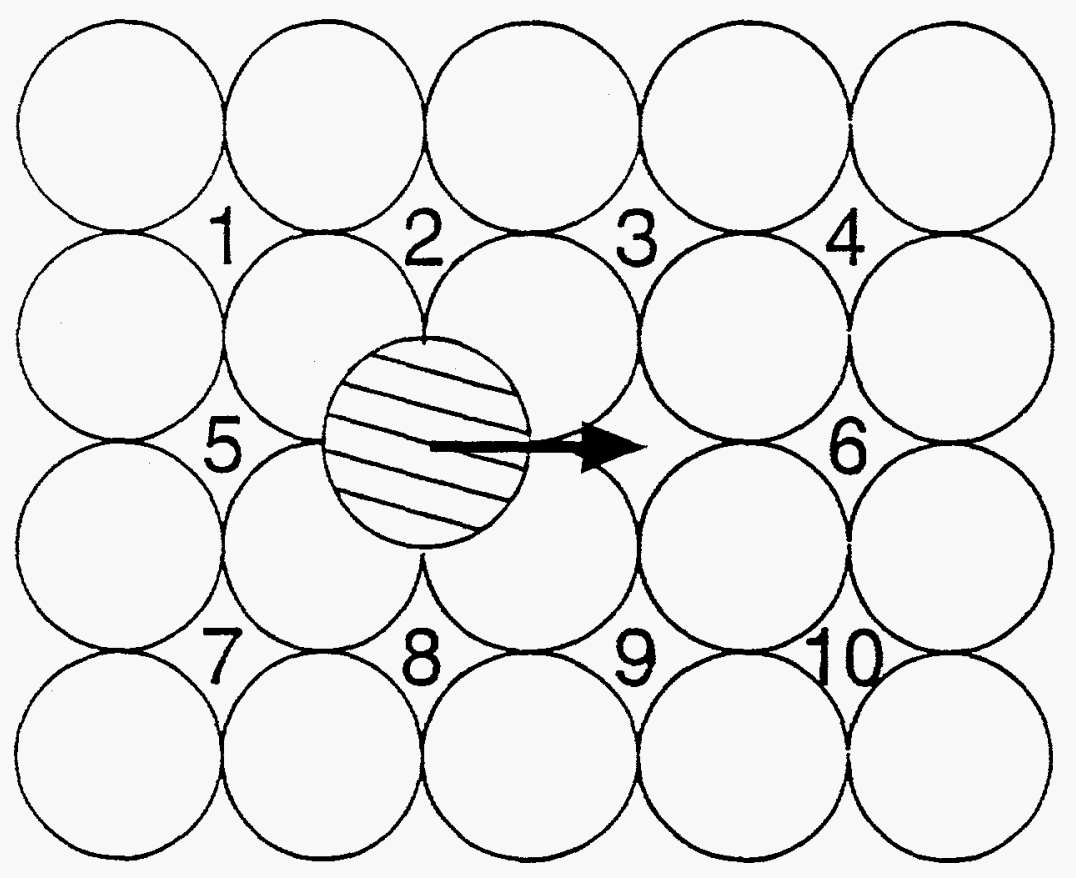

Figure 1. Rate catalog template for adatom hops on metal fcc(100) surface. Each of the ten sites can be empty or occupied, for a total of 210 possible environments affecting the hop rate. The catalog of these 1024 rates, computed using transition state theory, provide input for a kinetic Monte Carlo simulation. 


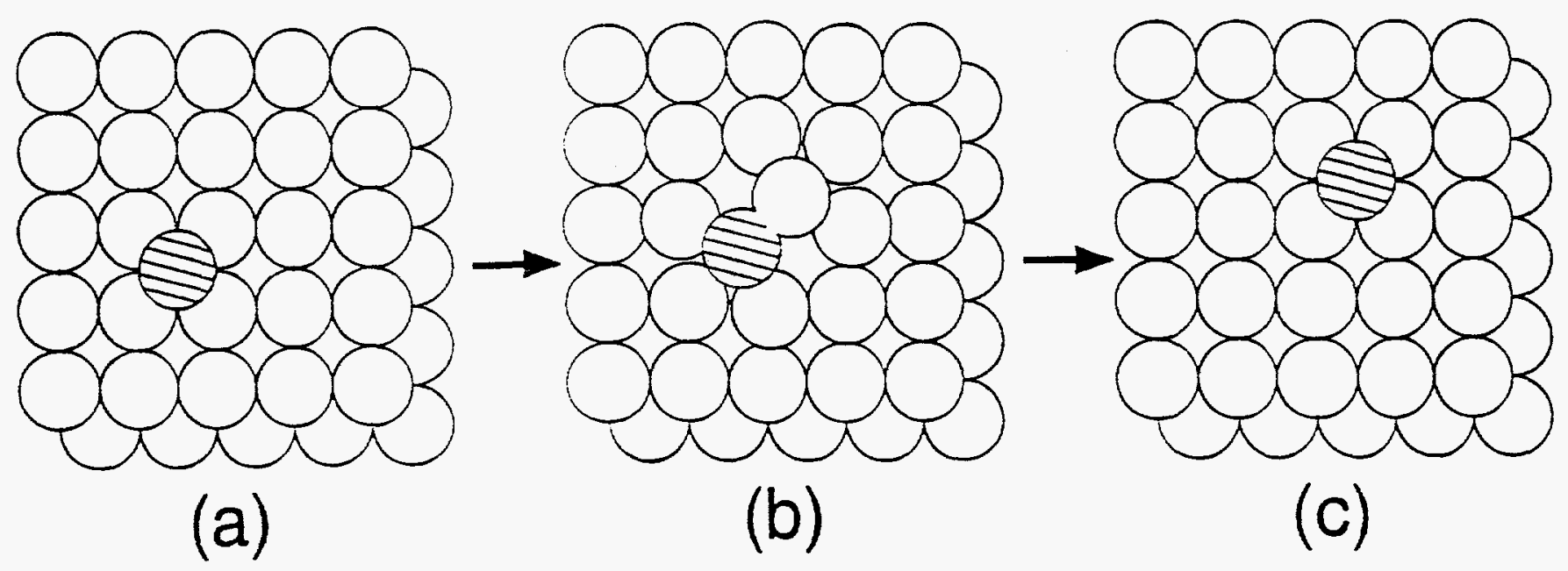

Figure 2. Exchange mechanism on metal fcc(100) surface. Until 1990, adatom diffusion on fcc $(100)$ was thought to occur solely by adatom hops, as indicated in Figure 1. This exchange event is now known to be the dominant diffusion mechanism for some fcc metals, including platinum, iridium and aluminum. 


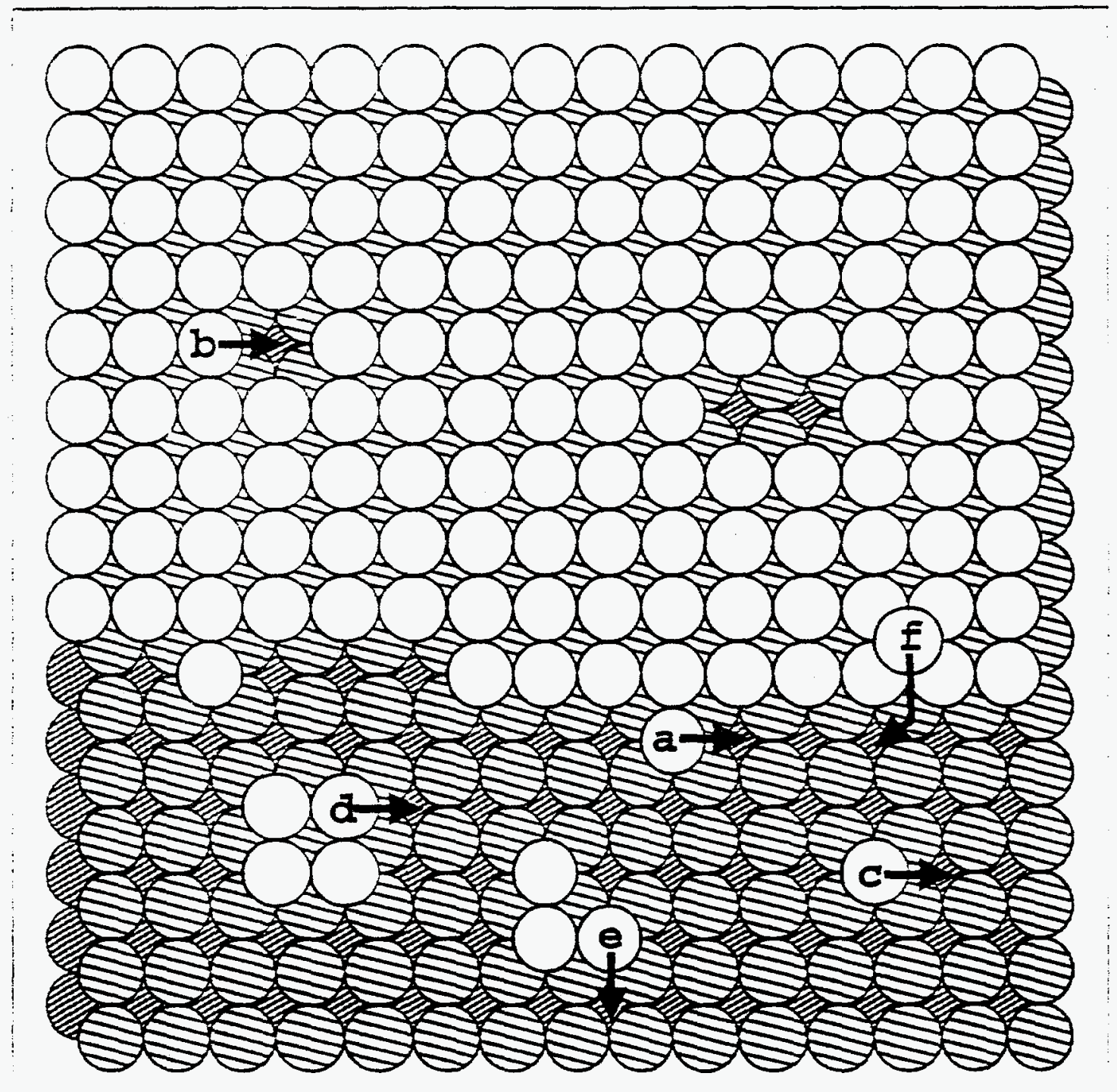

Figure 3. Schematic illustration of a metal fcc(100) surface during growth, showing the mechanisms that would be included in a kinetic Monte Carlo simulation prior to 1990. 


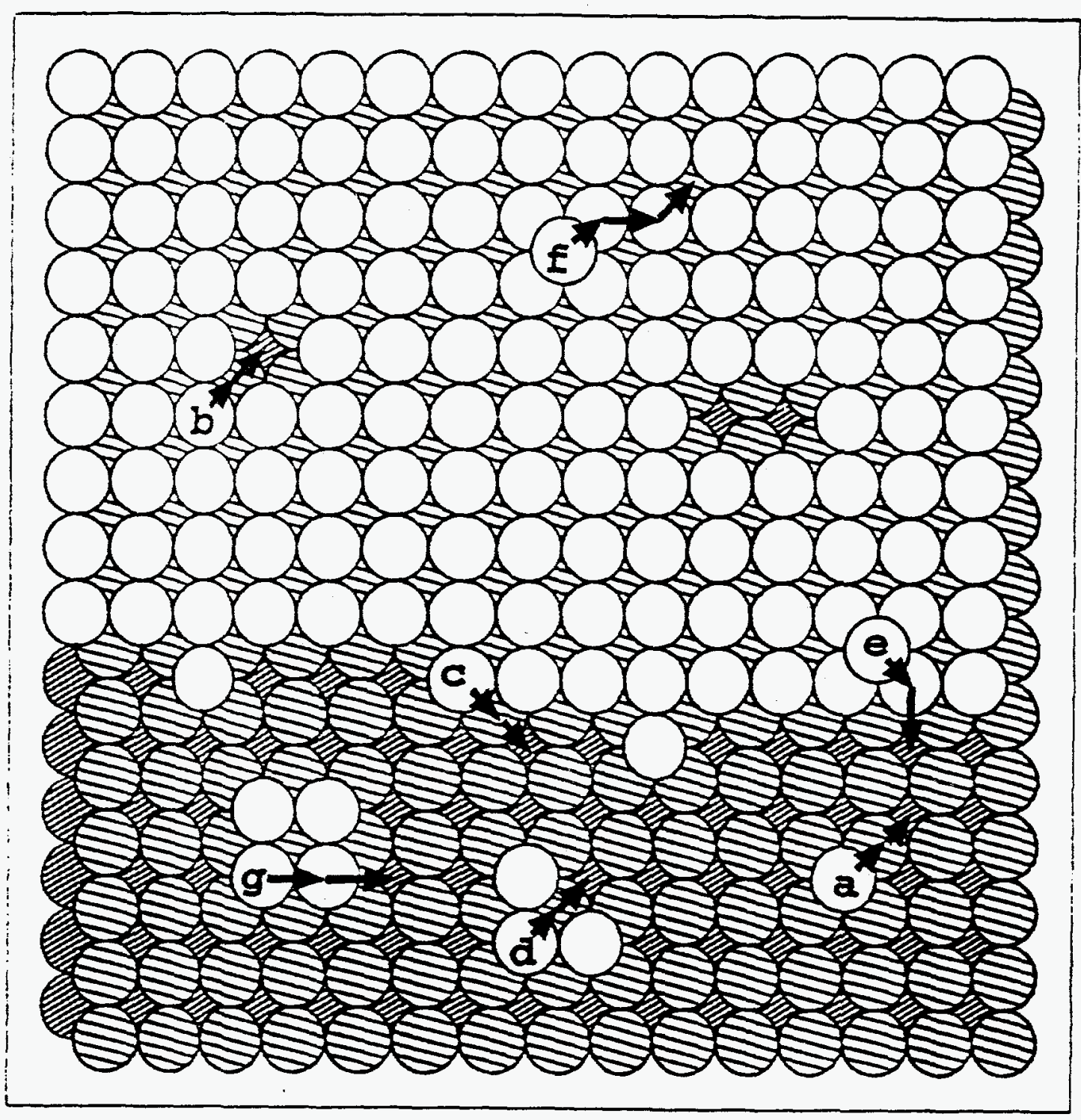

Figure 4. Essentially the same surface as in Fig. 3 (one adatom has been added), showing new mechanisms discovered since 1990. A proper dynamical treatment would require inclusion of all these. There are probably others not yet discovered. 


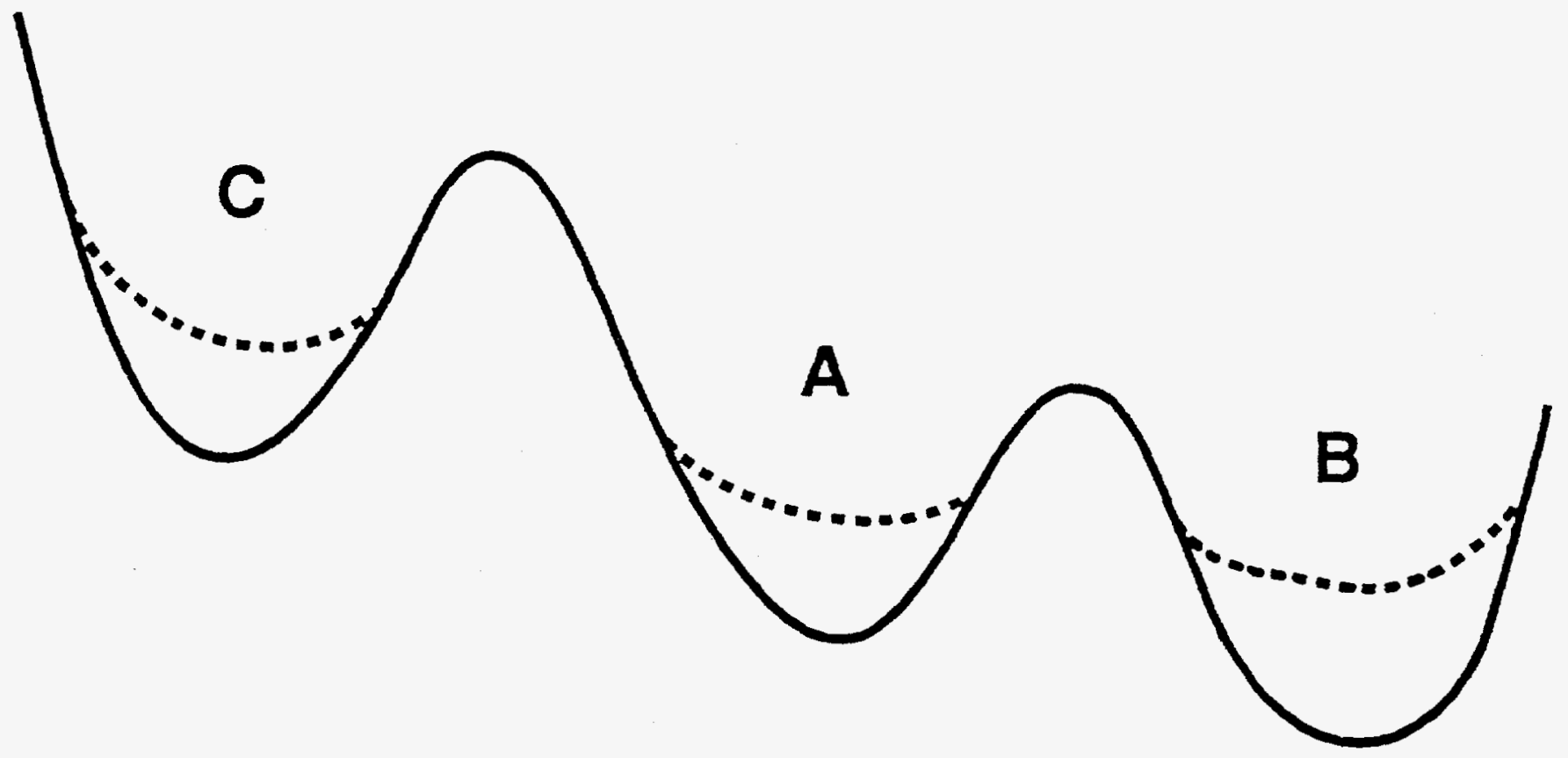

Figure 5. Illustration of the hyperdynamics method. A trajectory is evolved on the biased potential (dashed line) rather than the original potential (solid line). Relative escape probabilities are preserved because the bias potential is zero at the dividing surfaces. The accelerated time scale is calculated as the simulation proceeds. 

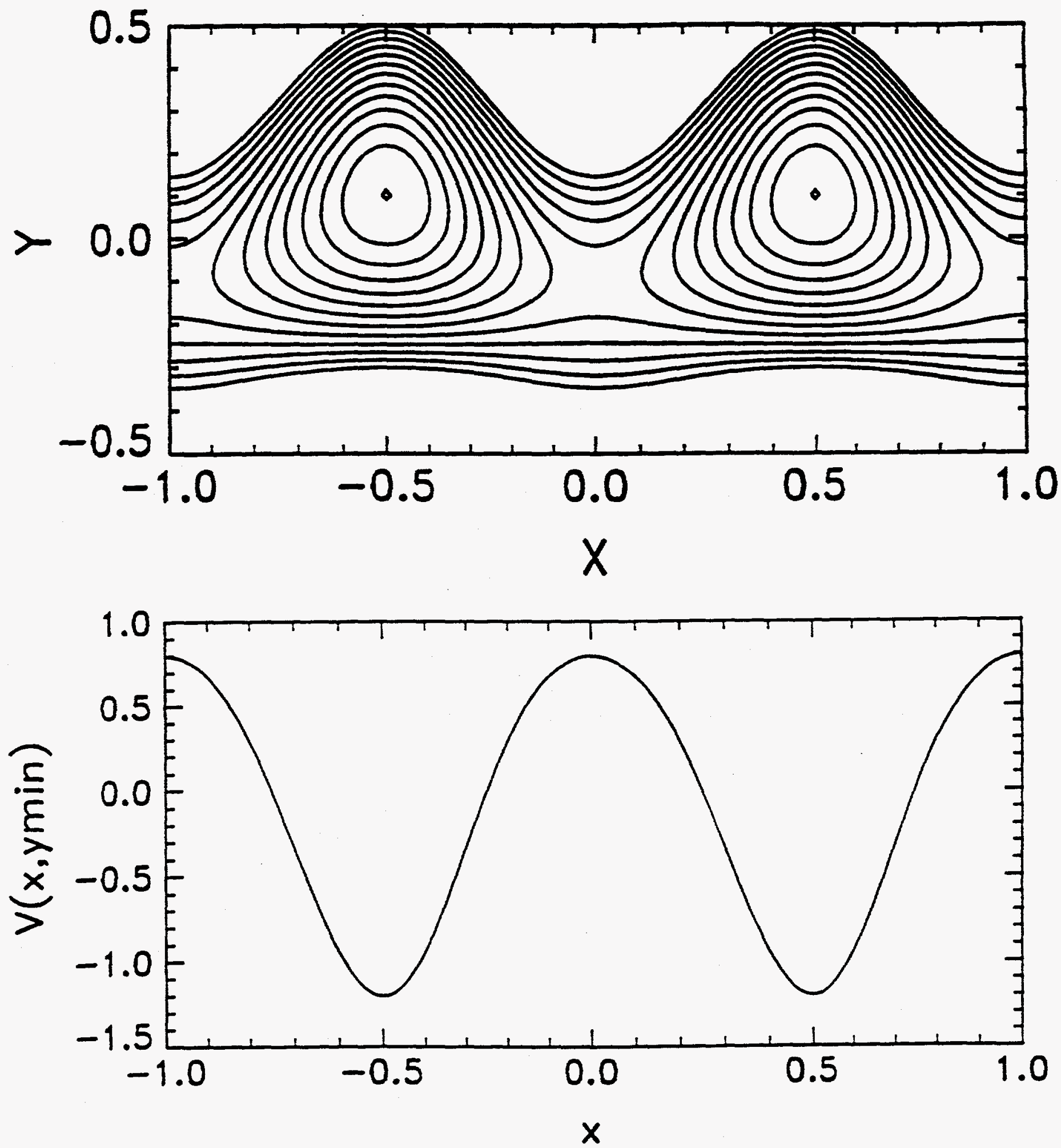

Figure 6. Two-dimensional model potential. The potential is periodic in the $\mathrm{x}$ direction and parabolic in the y direction. Two periods are shown. (a) contour plot; (b) projection into one dimension: minimum energy along $y$ for each value of $x$. 

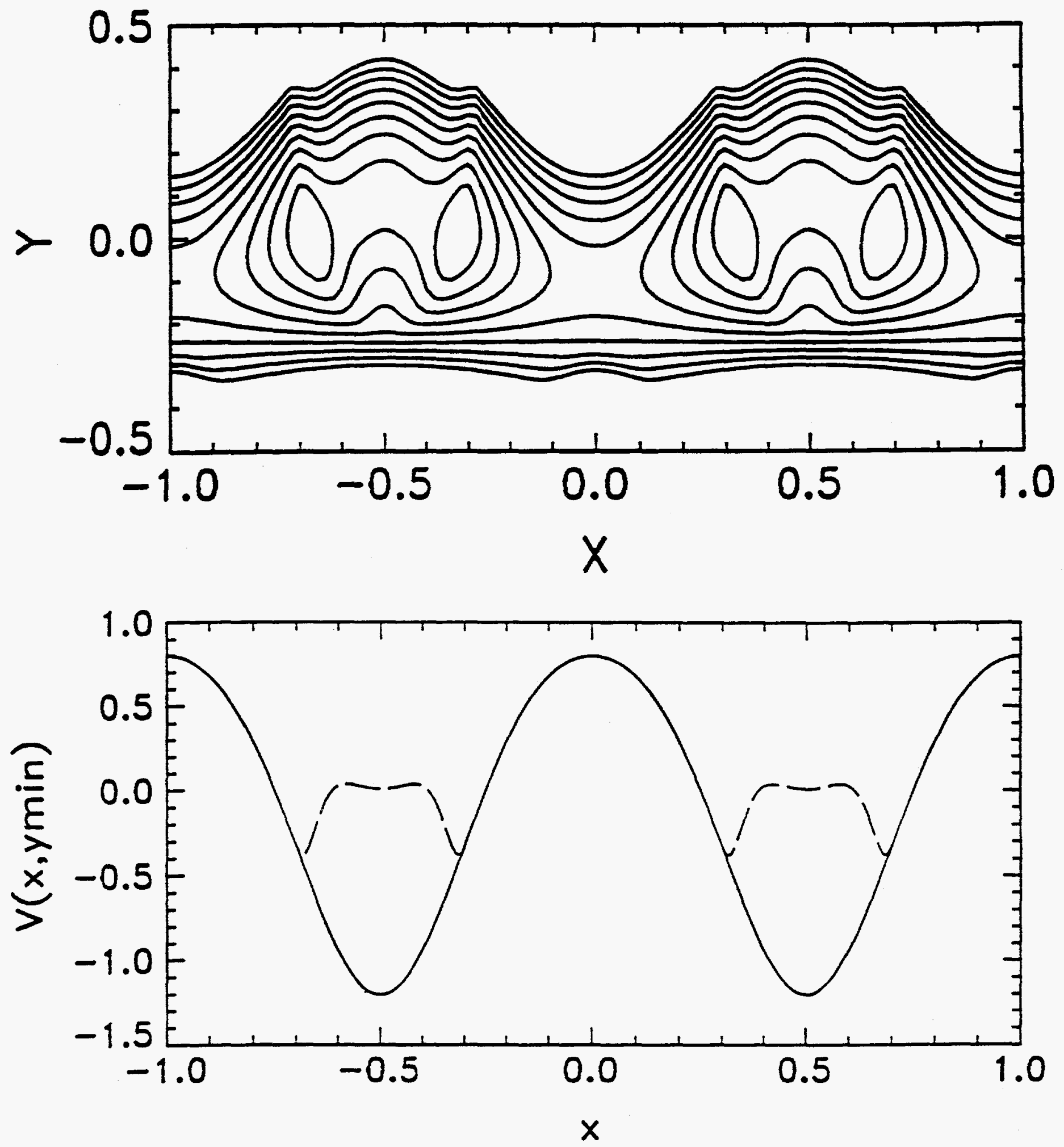

Figure 7. The potential described in Fig. 6, modified by a bias potential based on the lowest eigenvalue of the Hessian matrix. Note that the dividing surface (the $x=0$ line) is slightly corrupted. 


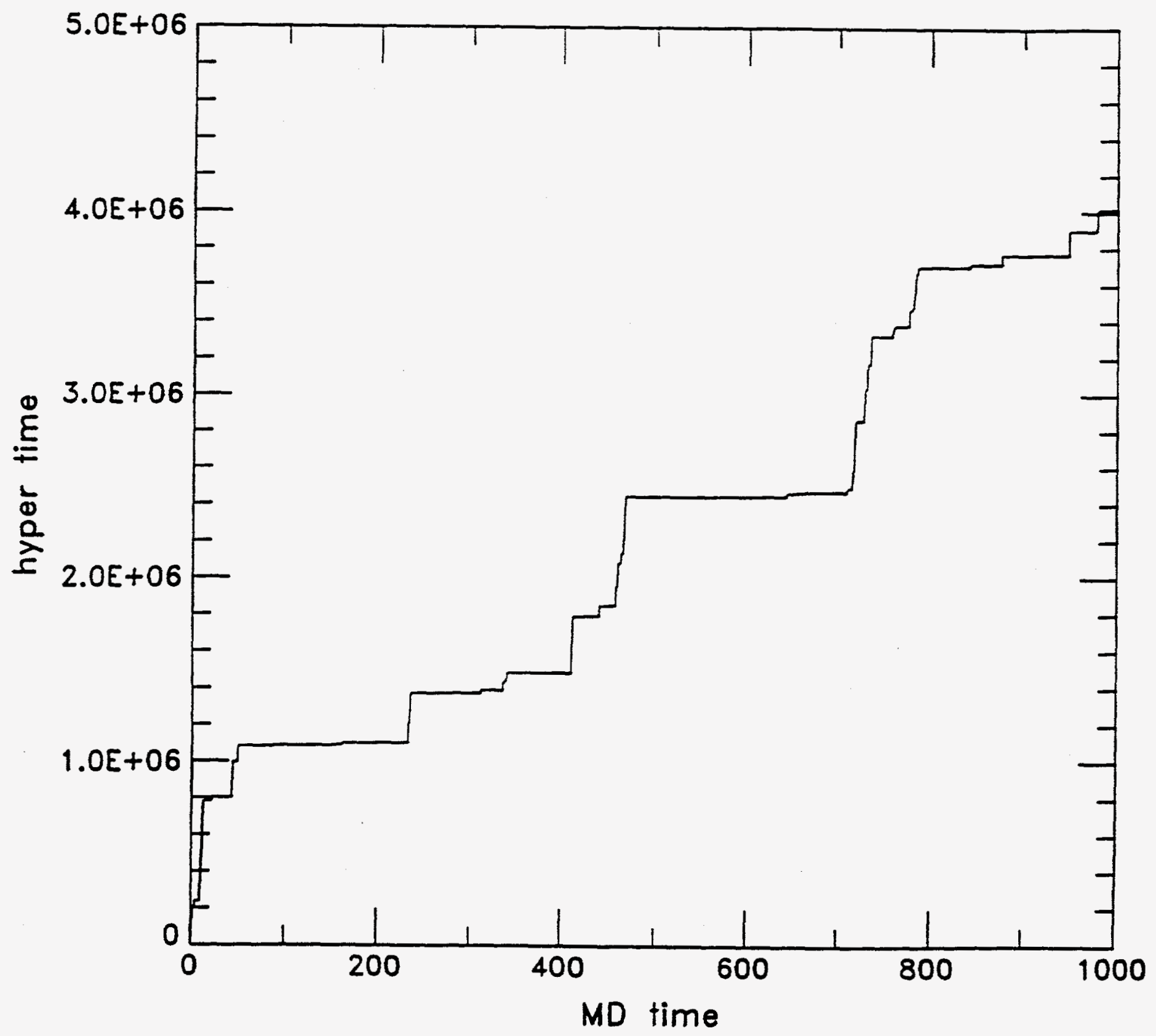

Figure 8. Evolution of the boosted time for a small section of the trajectory at $k T=0.1$. Both time and temperature are expressed in reduced units. The average boost factor is 3435 . 


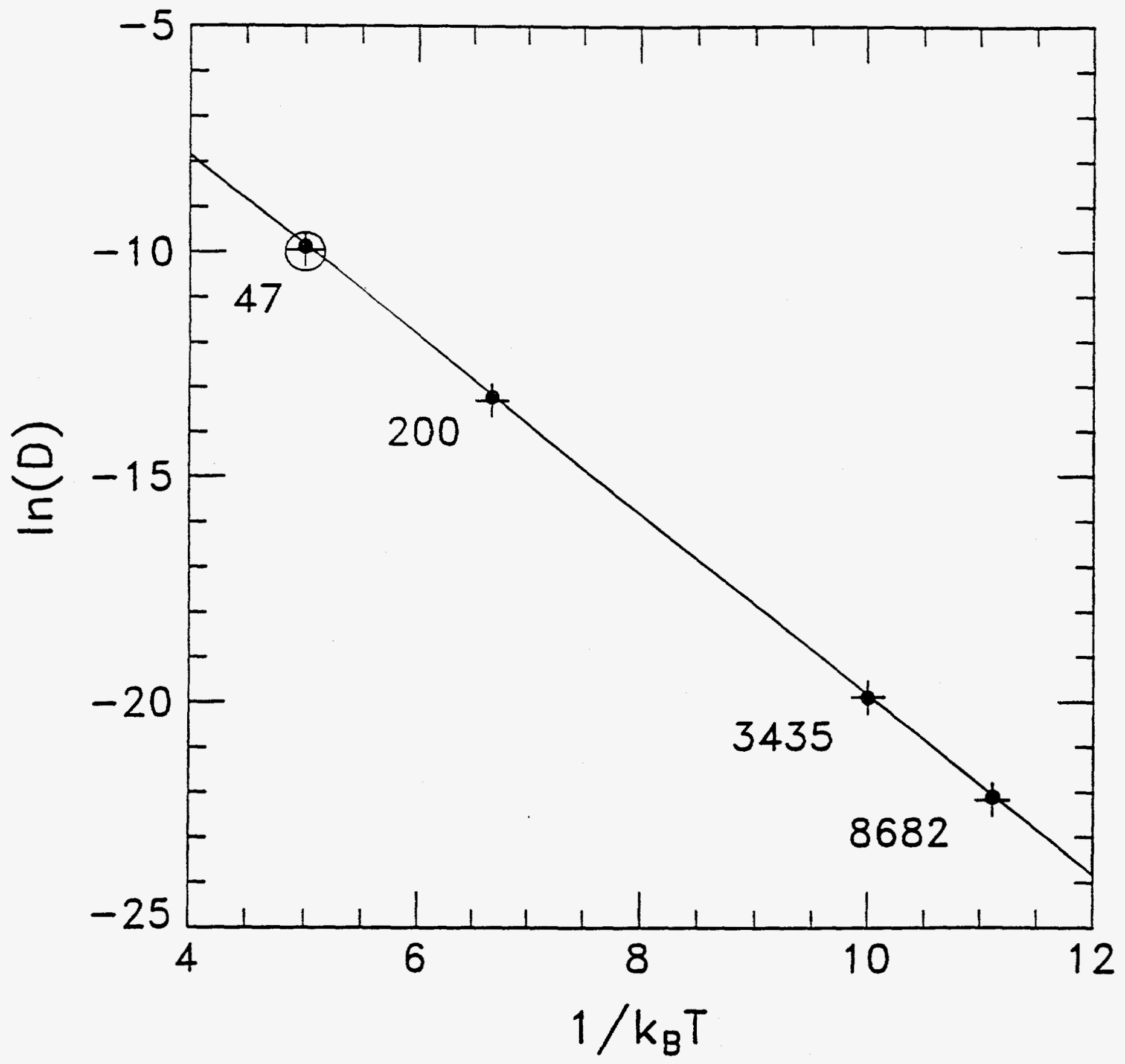

Figure 9. Arrhenius plot of diffusion constants computed for the 2-D potential shown in Figs. 6 and 7, using direct molecular dynamics (open circle), hyperdynamics (filled circles), transition state theory with dynamical corrections (plus symbols), and harmonic transition state theory (solid line). 


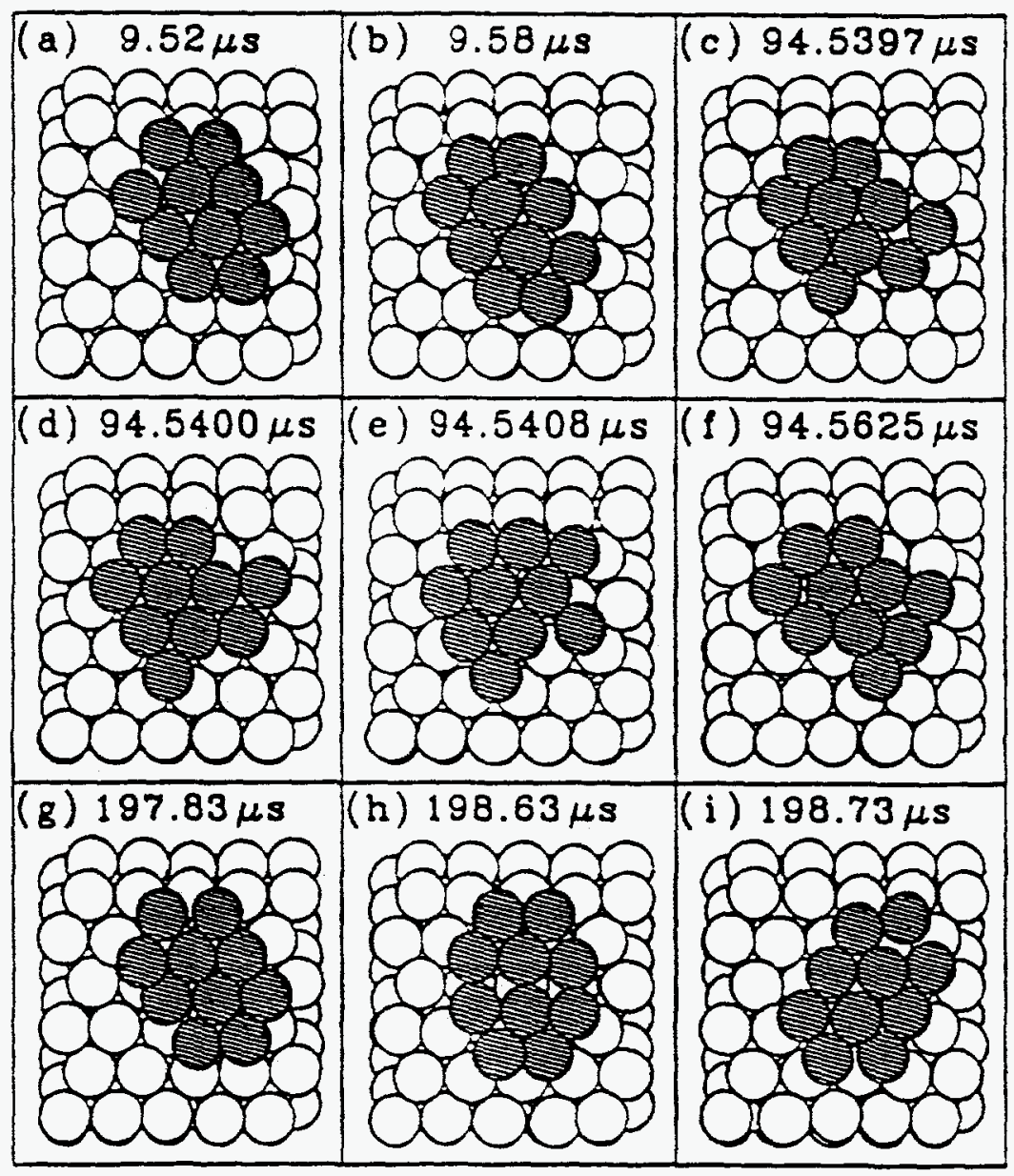

Figure 10. Selected snapshots from hyperdynamics simulation of 10-atom Ag cluster on $\mathrm{Ag}(111)$ surface. Three diffusive mechanisms can be observed, as discussed in text. Boosted time values are shown. 


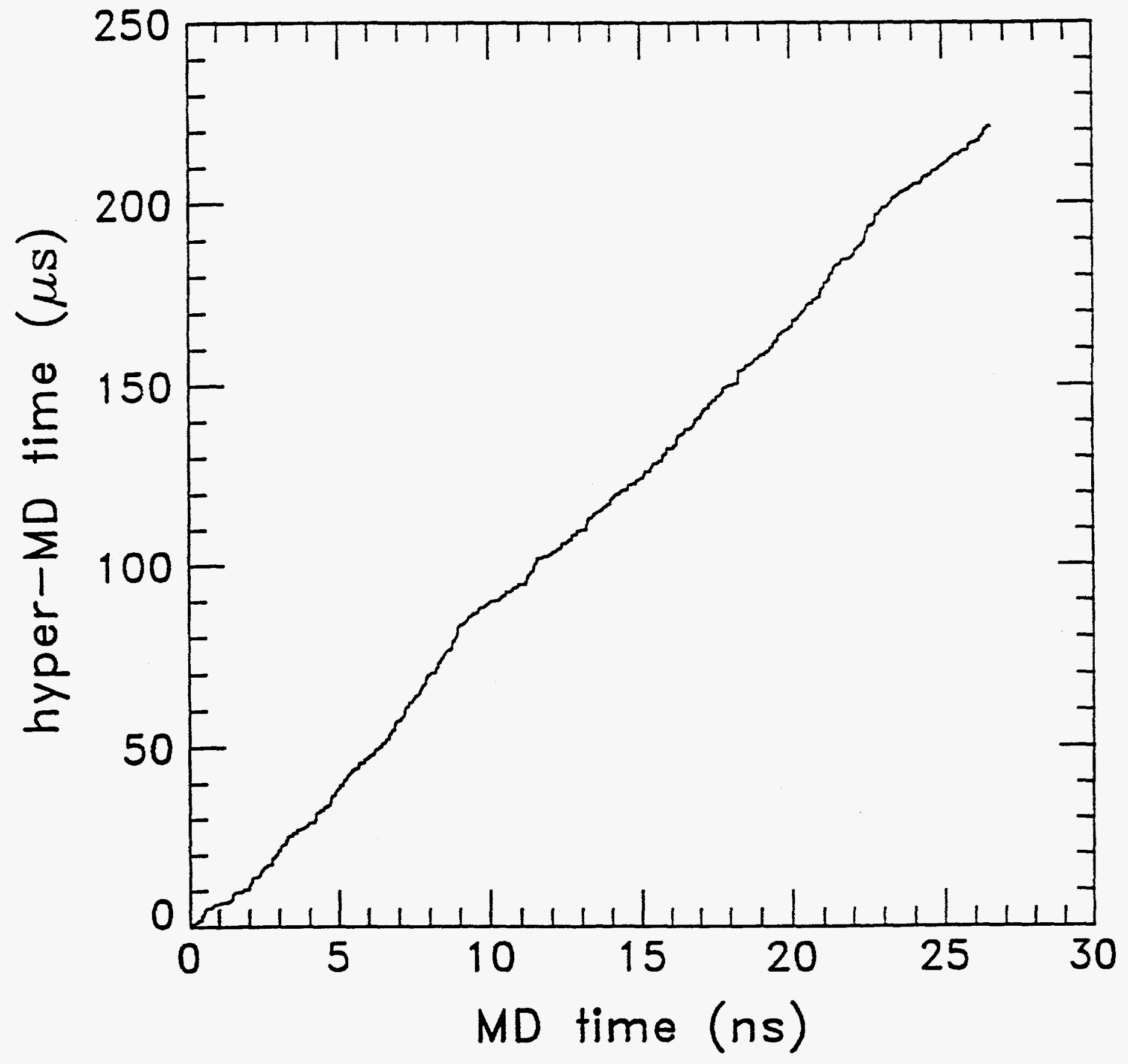

Figure 11. Evolution of the cumulative boosted time during the hyperdynamics simulation of a 10-atom Ag cluster on $\mathrm{Ag}(111)$. The average boost factor is 8310 . 


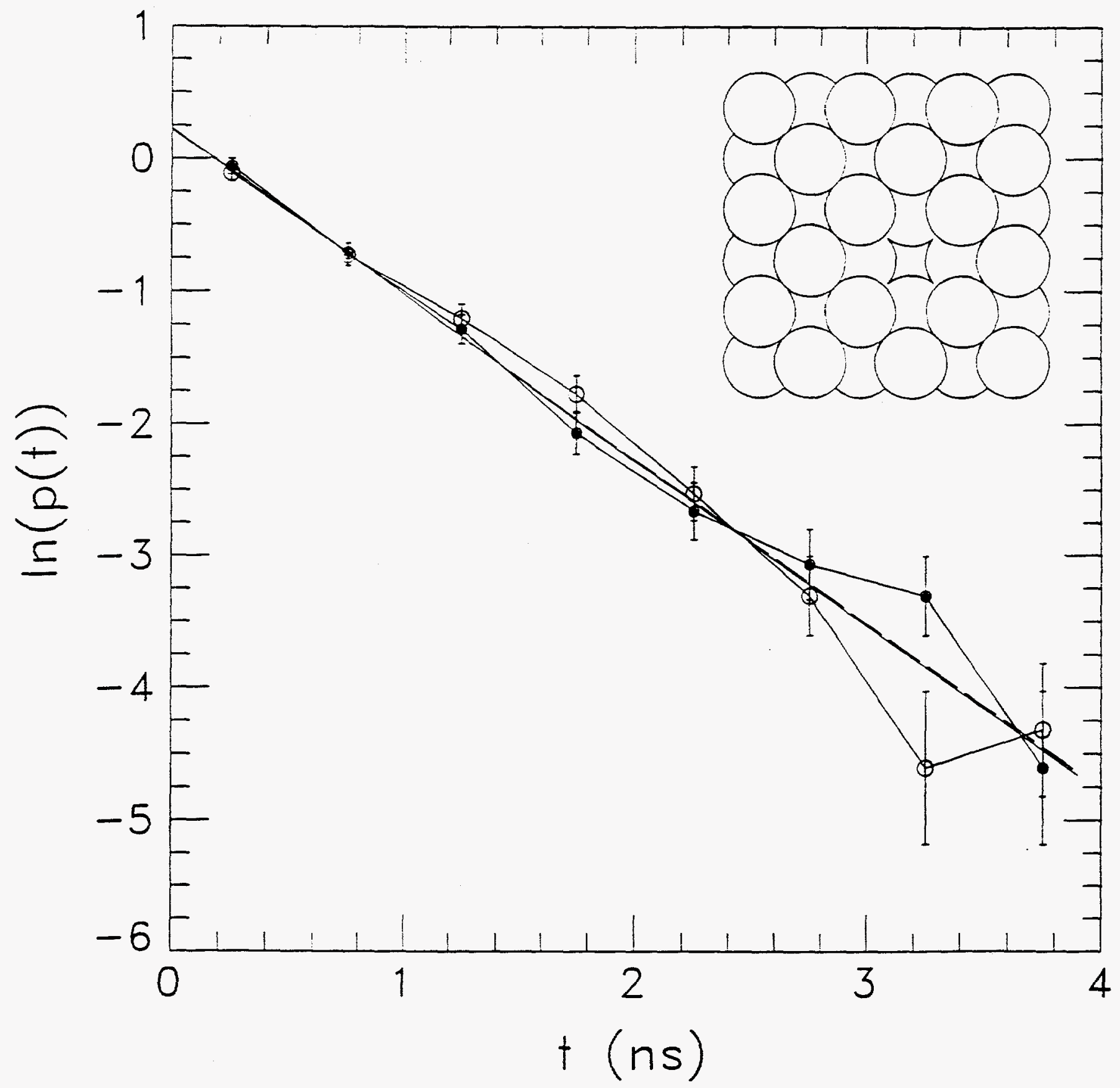

Figure 12. Escape time probability distribution for surface vacancy diffusion on $\mathrm{Cu}(100)$, obtained from a single trajectory (open circles, dashed line) and from the parallel-replica method (filled circles, solid line) implemented on 15 processors. The parallel efficiency in this case is over $90 \%$. 


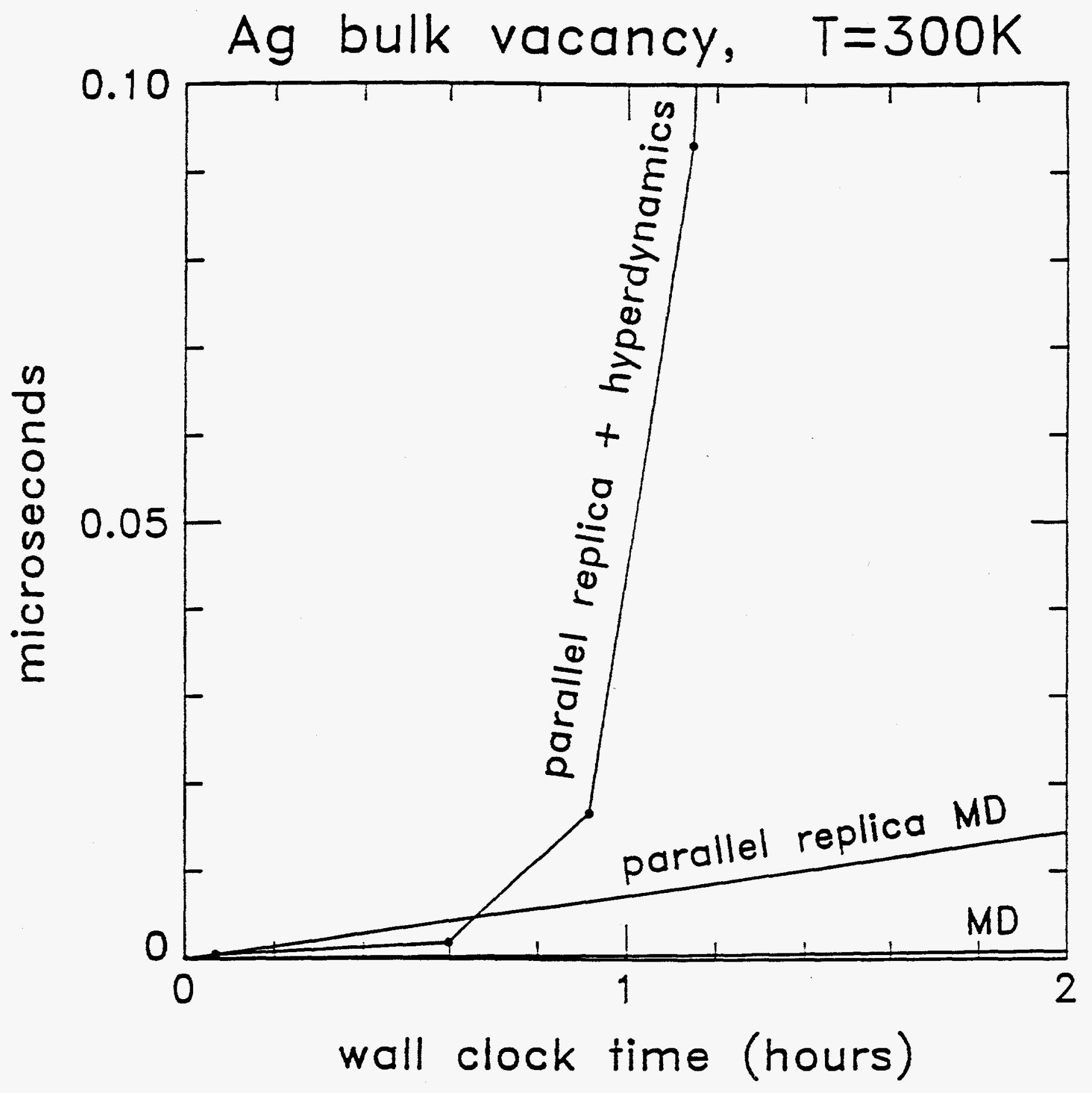

Figure 13. Time evolution during simulation of a bulk silver vacancy, combining the hyperdynamics method with parallel replica method. Only a fraction of the time leading up to the first vacancy hop is shown, as discussed in the text. The simulation achieved 1.9 milliseconds in 65 hours of wall clock time. 